\title{
EP-166
}

\section{Stool elastase as an independent prognostic factor in patients with pancreatic head cancer}

Honam HWANG, Hongbeom KIM*, Jae Seung KANG, Yoo Jin CHOI, Heeju SOHN, Jung Min LEE, Wooil KWON, Jin-Young JANG

Department of Surgery, Seoul National University Hospital, Seoul, Korea

Introduction: In pancreatic cancer, pancreatic exocrine insufficiency (PEI) is found in $44.5 \%$, is more common in pancreatic head cancer. Patients with PEI have an increased risk of malnutrition, which in turn increases morbidity and mortality. The aim of this study was to analyze the prognosis according to the PEI measured by Stool elastase (SE) level in patients with pancreatic head cancer.

Methods: Patients who underwent pancreaticoduodenectomy due to pancreatic cancer at Seoul National University Hospital from 2011 to 2015 were included. Among these patients, the patients who had the preoperative SE level were analyzed. Groups were classified based on $100 \mu \mathrm{g} / \mathrm{g}$, which can be defined as severe PEI.

Results: Total 143 patients were included. Median preoperative stool elastase level was $67.2 \mu \mathrm{g} / \mathrm{g}$. 84 patients (58.7\%) were in the low SE group and 59 patients (41.3\%) were in the high SE group. There were no significant difference in R0 resection rate, final pathologic stages and adjuvant treatment between the two groups. The two groups had significantly different overall survival (OS) and disease-free survival (DFS), and low SE group had a worse prognosis (median OS: 17 versus 25 months, $p=0.035$, median DFS: 8 versus 14 months, $p=0.006$ ). On multivariate analysis, stool elastase $\geq 100 \mu \mathrm{g} / \mathrm{g}$ (hazard ratio (HR): $1.487, p=0.048$ ), and no lymph node metastasis (HR: 1.852, $p=0.005$ ) were found to be independent prognostic factors for better OS. And those were also significant prognostic factor for DFS.

Conclusions: PEI measured by SE level is an independent prognostic factor for patients with pancreatic head cancer who undergoing pancreaticoduodenectomy. In these patients, management of severe PEI such as nutritional support is expected to be required. 